\title{
An extensive study of the photometric behaviour of RV Tauri variables
}

\author{
E. Paunzen ${ }^{1}$, I.L. Andronov ${ }^{2}$, L.L. Chinarova ${ }^{2}$, M. König ${ }^{1}$, M. Rode-Paunzen ${ }^{1}$ \\ 1 Institut für Astronomie, Türkenschanzstr. 17, 1180 Vienna, Austria \\ 2 Department of Astronomy and Astronomical Observatory, Odessa National University, T.G. Shevchenko \\ Park, Odessa 65014, Ukraine
}

\begin{abstract}
The project of time series analysis for the photometric observations of a representative group of RV Tauri type stars is described. This class of variables consists of about 100 supergiants in the Milky Way. Their light curves show in general two unequal minima with several individual features which are not explained by now. The complementary methods of periodogram, scalegram and wavelet analysis as well as statistically optimal fits have been applied. This research will result in an atlas of the light curves within the time and phase domains. In addition, a catalog of the observational characteristics of variability including the time and magnitude of the extrema for the program stars will be computed. Statistical correlations between these characteristics will give a strict observational test for the theoretical models explaining the RV Tauri phenomenon.
\end{abstract}

\section{Introduction}

The class of RV Tauri variables comprises about 100 supergiants in the Milky Way. Their light curves show two unequal minima which makes the decision about the physically significant period rather uncertain, i.e. the interval between successive maxima or minima (single period), or the interval between successive deep minima (double period). The period from one deep minimum to the next ranges from 30 to 150 days. There are two subgroups, the RVa objects which maintain an approximately constant mean brightness and RVb which exhibit long-term periodicities of up to 2500 days. An overview of observations and the analysis of such light curves can be found in Percy et al. (2003).

They have spectral types from $F$ to $G$ at maximum light and $K$ to $M$ at minimum. In the Hertzsprung-Russell-Diagram they are executing blue loops from the asymptotic giant branch (AGB), or are post-AGB stars which makes them interesting objects for the theoretical stellar evolution theory.

Still, the nature of the found variability, the precise evolutionary status and an explanation of the RVb phenomenon is not known. The most excepted theory is the double-mode pulsator model in which the period ratio is more or less two to one (Takeuti \& Peterson 1983). In principle, the double period is due to the fundamental whereas the single period is the first overtone mode. Both are excited if the effective temperature of a star is below a certain critical value. The ratio of these two modes is, as observed, two to one according to the theoretical pulsation models (Fokin 1994).

There seems to be a connection between RV Tauri variables with Type II Cepheids as well as yellow semiregular (SRd) variables. However, these are only puzzles by now and no definite answer has raised up to now. 


\section{Overview and aims of our project}

We have collected all published and available photometric data of known galactical RV Tauri variables. Since these objects have very deep minima and maxima, they are also observed by the large amateur community. If available, we have also included these archives. For some stars, we have used photographic observations from the Odessa plate collection. The first published data date back to 1890 , so for some objects (e.g. $\mathrm{V} \mathrm{Vul),} \mathrm{time} \mathrm{basis} \mathrm{of} \mathrm{more} \mathrm{than}$ 100 years have been achieved. This means that even the long-term periodicities of up to 2500 days are very well covered. In total, more than 120 different publications have been included in our analysis.

One of the most crucial points of the analysis is to homogeneous the data spanning observational techniques and instruments of more than 100 years.

Our final goal is to analyse the RVa and RVb subgroups separately. The precise knowledge of all relevant pulsational parameters for all time basis is essential to understand the physical processes acting in this group of variable stars.

\section{Time series analysis}

RV Tauri type stars show a complex temporal behaviour, thus the time series analysis should be resulted both in the average parameters of variability, which characterize double-peak structure of the light curve as well as long-term waves, and in the analysis of the cycle-tocycle variability. For this purpose, the following methods have been applied:

- Periodogram analysis using the sinusoidal approximation with the program "Four" (Andronov 1994).

- Determination of the corrected value of the period (as well as amplitudes and phases) using the trigonometric polynomial fit with a statistically optimal value of the degree of mathematical model (program "FDCN" described in Andronov 1994).

- Scalegram analysis using the $\sigma(\Delta t)$ and $\Lambda(\Delta t)$ test-functions (Andronov 1997, 2003) to determine statistically optimal value of the filter half-width $\Delta t$ and thus the best "running parabola" fit for the light curve.

- Wavelet analysis based on the weighted least squares extension of the method to the case of non-homogeneously distributed times of observations (Andronov 1998, 1999), which allows to study variability of the shape of the light curve with a time scale of few pulsation periods. An example of the application of the method to the RV Tauri type star DF Cygni can be found in Andronov (1999).

- "Running sine" analysis (Chinarova 1998, Andronov 1999, 2003), which results in the time dependency of the period-averaged mean value, amplitude and phase of the pulsations with a relatively stable period.

- Determination of times and magnitudes of the individual extrema using either the "asymptotic parabola" (Marsakova \& Andronov 1996), or "best timing polynomial" (Chinarova \& Andronov 2000) fits.

\section{Achieved results}

The time series analysis of the photometric observations of long-period pulsating variables using these methods have been applied to 53 Mira-type (Marsakova \& Andronov 1998) 
and 173 semi-regular (Chinarova \& Andronov 2000) stars with corresponding catalogues of characteristics presented in these papers and the discussions published elsewhere.

The current research extends such a study to the case of RV Tauri type stars. It will result in:

- An atlas of the light curves for the studied objects including compiled published photometric data with filtering ("evaluation", according to the AAVSO terminology), i.e. the statistically optimal fits within the time and phase domain.

- A catalogue of the observational characteristics of the mean light curves, including the period, amplitudes and initial epochs for contributions with different periods; values of the periods and possible quasi-periodic oscillations obtained using different methods of time series analysis.

- A catalogue of the individual characteristics of the maxima and minima.

- Statistical study of the relations between all different characteristics.

Acknowledgments. This research was performed within the project P17580 of the Austrian Fonds zur Förderung der wissenschaftlichen Forschung (FWF) with a partial support of the Ministry of Education and Science of Ukraine. We have used the international databases of the photometric observations published by the AFOEV and the VSOLJ.

\section{References}

Andronov, I. L. 1994, Odessa Astron. Publ. 7, 49 (http://il-a.pochta.ru//oap7_049.ps.z)

Andronov, I. L. 1997, A\&AS 125, 207

Andronov, I. L. 1998, Kinem. Phys. Celest. Bodies 14, 6, 490

Andronov, I. L. 1999, in: V. B. Priezzhev, V. P. Spiridonov (eds.), Self-Similar Systems, Dubna, Russia, Joint Inst. Nucl. Res., 57 (http://il-a.pochta.ru/dubna.ps.z)

Andronov, I. L. 2003, ASP Conf. Ser. 292, 391

Chinarova, L. L. 1998, in: J. Dusek, M. Zejda (eds.), Proc. 29th Conf. Variable Star Res., Brno, Czech Republic, 38 (http://il-a.pochta.ru/brno8.ps)

Chinarova, L. L., \& Andronov, I. L. 2000, Odessa Astron. Publ. 13, 116 (http://il-a.pochta.ru/oap13_116_text.htm)

Marsakova, V. I., \& Andronov, I. L. 1996, Odessa Astron. Publ. 9, 127 (http://oap09.pochta.ru)

Marsakova, V. I., \& Andronov, I. L. 1998, Odessa Astron. Publ. 11, 79 (http://il-a.pochta.ru/wj/oap11_79.htm)

Fokin, A. B. 1994, A\&A 292, 133

Percy, J. R., Hosick, J., Leigh, N. W. C. 2003, PASP 115, 59

Takeuti, M., \& Petersen, J. O. 1983, A\&A 117, 352 